Grupos temáticos de la Red Internacional de Historiógrafos de la Comunicación Fortalecer la investigación desde el enfoque interdisciplinario

Dariel Mena Méndez, Clara Victoria Meza Maya, Yamilé Ferrán Fernández Improntas de la comunicación y la cultura (N. $\left.{ }^{\circ} 6\right)$, eozo, 2018 ISSN 2469-0457 | https://doi.org/10.24215/24690457eo3o http://perio.unlp.edu.ar/ojs/index.php/improntas

FPyCS | Universidad Nacional de La Plata La Plata | Buenos Aires | Argentina

\title{
Grupos temáticos de la Red Internacional de Historiógrafos de la Comunicación
}

\author{
Fortalecer la investigación desde el enfoque interdisciplinario \\ Thematic Groups of the International Net of Historiographers \\ of the Communication \\ To Strengthen the Investigation from the Multidisciplinary Perspective
}

\section{Dariel Mena Méndez}

darielito.mena@gmail.com

https://orcid.org/0000-0003-0640-0446

Universidad de Cienfuegos | Cuba

\section{Yamilé Ferrán Fernández}

yferran@fcom.uh.cu

https://orcid.org/0000-0002-1698-0678

Universidad de La Habana | Cuba

\section{Resumen}

En el presente trabajo se describen los criterios que guiaron la conformación de los grupos temáticos de la Red Internacional de Historiógrafos de la Comunicación, una organización académica de investigación constituida en 2016, en línea con la estrategia seguida en los últimos años por múltiples organizaciones profesionales y académicas para impulsar los diálogos inter y transdisciplinares.

Palabras clave

Historiografía, comunicación, investigación, grupos temáticos

\section{Abstract}

This paper describes the criteria that guided the formation of the thematic groups of the International Network of Historiographers of Communication [Red Internacional de Historiógrafos de la Comunicación], an academic research organization constituted in 2016, as a strategy followed in recent years by multiple professional and academic organizations to promote inter and transdisciplinary dialogues.

Keywords

histograph, comunication, research, thematic groups 


\section{Grupos temáticos de la Red Internacional de Historiógrafos de la Comunicación}

Fortalecer la investigación desde el enfoque interdisciplinario

Por Dariel Mena Méndez, Clara Victoria Meza Maya y Yamilé Ferrán Fernández

\section{Introducción}

Referirse a líneas o a ejes y grupos temáticos (ET/GT) de investigación en comunicación requiere aludir a tres aspectos fundamentales: en primer lugar, a los intereses epistémicos, teóricos y metodológicos que en la actualidad constituyen tendencia a nivel internacional, en las comunidades académicas regionales o en los ámbitos educativos nacionales cuyos centros de enseñanza cuentan con programas de comunicación social, periodismo, ciencias de la información y otras disciplinas afines; en segundo lugar, a las problemáticas que afrontan las sociedades contemporáneas; $y$, por último, a la impronta que ha significado lo multidisciplinar en la construcción epistémica del objeto de estudio.

No podemos pensar en la comunicación desestimando cuánto aportan la historia, la política, la economía, la sociología, la antropología, la psicología y la filosofía. Desde sus inicios, a mediados de la pasada centuria, la participación de estas y de otras disciplinas en la edificación del corpus teórico de la comunicación, no solo ha favorecido que los fenómenos y las prácticas massmediáticas sean examinados desde la lógica multidisciplinaria, sino que también ha propiciado la diversificación de objetos, de categorías y de dimensiones de estudio que durante mucho tiempo habían sido examinadas desde otras regiones de pensamiento. Sin embargo, es notable el privilegio que se ha otorgado durante décadas a la investigación sobre los mass media, demostrándose un descuido en cuanto a la visualización de otros fenómenos de comunicación que tributan a la categoría de producción simbólica en su concepción integral.

Hablar de multidisciplinariedad / interdisciplinariedad en ciencias de la comunicación, no solo admite que se revelen y se interpreten los aportes conferidos por otras disciplinas, sino también que se entienda el carácter diverso y plural que distingue a los discursos investigativos de los últimos años. 
Es aquí donde desempeñan un rol importante las universidades, los centros de estudio y las redes y asociaciones académicas de investigación en comunicación, algunas con carácter más general y otras que se definen como organizaciones especializadas, entre las que se destacan: la Red de Investigación en Comunicación Comunitaria, Alternativa y Participativa (RICCAP), la Red Iberoamericana de Investigadores en Publicidad, la Asociación Española de Estudios de Mercado, Marketing y Opinión (AEDEMO), la Asociación para la Investigación de Medios de Comunicación de España (AIMC), la Asociación de Investigadores en Relaciones Públicas (AIRP) y la Asociación de Historiadores de la Comunicación (AHC), ambas de España.

Todas ellas surgen como alternativa para favorecer el intercambio académico y para contribuir a la solución de las problemáticas que lastra la cimentación de la ciencia de la comunicación en cuanto a los nichos de investigación, para lo cual se diseñan estrategias y políticas científicas que otorgan un sentido holístico y plural al campo epistemológico mediante líneas de estudio, grupos y ejes temáticos. En este marco, los diferentes congresos, seminarios y simposios promovidos por estas instituciones se constituyen como espacios de cooperación, de socialización y de intercambio científico por excelencia.

Sin embargo, la pesquisa en estatutos, en reglamentos y en catorce convocatorias públicas comprendidas entre 2010 y 2017 demostró algunos ejes temáticos poco favorecidos en los eventos y en las plataformas de investigación internacionales. Los estudios empíricos sobre procesos y sobre prácticas comunicativas y culturales no massmediáticas que se producen en el espacio público, la producción infocomunicativa de los movimientos sociales y populares, y los estudios históricos / historiografía de la comunicación, figuran entre los mencionados temas.

Como reconocen varios autores, la enseñanza y la investigación en historia de la comunicación continúa siendo un nicho en las escuelas y en las facultades de comunicación, fundamentalmente en Latinoamérica.

A pesar de la constatación de que el proceso de conformación autónoma de la historia de la comunicación se da en un contexto de renovación epistemológica, no es el histórico el paradigma que más pesa dentro de las corrientes investigadoras de la comunicación [...]. Quizás una manera de entender el poco peso de lo histórico en el magma de la investigación en comunicación sea que, durante el proceso de dignificación del campo comunicativo, la interdisciplinariedad de la perspectiva histórica la haya dejado un tanto de lado en unas escuelas y otras, entre los estudios culturales y la más amplia perspectiva crítica (Ferré Pavía, 2008, pp. 2-3). 
Lo referido pudo corroborarse en una entrevista realizada al presidente de la AHC, Antonio Laguna Platero (2016), quien expuso que «continúa siendo este campo de los menos representativos de Iberoamérica, tanto en la docencia como en la investigación».

Constituyen estos los antecedentes y los motivos que dieron lugar a la fundación de la Red Internacional de Historiógrafos de la Comunicación (RIHC), el 28 de octubre de 2016 durante el VI Encuentro Iberoamericano de Comunicación que tuvo lugar en Santo Domingo, República Dominicana.

En tal sentido, en el presente artículo se propone dar respuesta a los siguientes interrogantes: ¿Cuáles son los grupos temáticos de la RIHC? ¿A qué lógicas responden dichos temas? ¿Cómo se conformaron? ¿Cuáles son sus perspectivas fundamentales? ¿Qué importancia se le atribuye a la construcción colectiva y a lo interdisciplinar en el proceso de constitución y de desarrollo de los GT de la red?

Para ello, el trabajo se inicia con algunas reflexiones sobre la perspectiva interdisciplinaria y sus nexos con las líneas de investigación en comunicación que se privilegian en los últimos años en Iberoamérica. Como antecedentes, nos acercamos a los GT de varias organizaciones académicas de investigación, apoyándonos en la revisión de diferentes estatutos de redes y de asociaciones, así como a un conjunto de convocatorias públicas a congresos, a simposios y a eventos internacionales.

\section{Interdisciplinariedad y líneas de investigación en comunicación}

Los enfoques disciplinariedad, interdisciplinariedad y transdisciplinariedad en los ámbitos educativos e investigativos adquieren legitimidad desde inicios del siglo XX, si bien es cierto que se reconocen antecedentes a finales del XIX con las primeras iniciativas de estudios integrados sobre educación. Se trata de la combinación de conocimientos que proceden de distintas regiones de pensamiento para la delimitación y la solución de una problemática específica. Para el profesor Gustavo León Duarte (2015), «implica necesariamente el trabajo grupal o el trabajo en y con un equipo de investigación integrado por distintas opciones disciplinares y en función de los objetivos de investigación [...]» (p. 4). 
En su proceso de construcción teórica, ha sido la comunicación una de las ciencias que más se ha nutrido de este tipo de ejercicio integrado. «El trabajo cooperado de las diferentes disciplinas [...] emerge como una necesidad debido a la complejidad de su objeto de estudio» (Dávila Lorenzo \& Lorenzo Suárez, 2016, p. 64).

Para Gabriel Lingeri (2013), el ensanchamiento de enfoques pluralistas y relativistas configuró la idea de la comunicación como interdisciplina y transdisciplina, «desde que se estableció - a mediados del siglo pasado - un solapamiento entre cultura y artefactos comunicacionales» (en línea).

En la actualidad se habla de comunicación desde discursos que la enmarcan como práctica, como producción y como proceso que transversaliza cada espacio en el cual los sujetos sociales construyen sus relaciones históricas y cotidianas. Por eso, las agendas de investigación se mueven en ámbitos diversos, a saber: los medios, las instituciones, las comunidades, el campo académico / investigativo, etcétera.

Adicionalmente, algunas líneas han adquirido mayor atención durante los últimos años: comunicación política, comunicación intercultural, estudios sobre el mensaje periodístico, de recepción y consumo, estudios sobre nuevas tecnologías y comunicación hipermedia, papel de la comunicación y la cultura en la construcción de identidades colectivas, comunicación organizacional y su implicancia económica, impacto de los sistemas sociales en el desarrollo de la comunicación pública, procesos y modelos de comunicación emergentes, comunicación para el desarrollo, educomunicación, estudios teóricos y aportes al campo académico de la comunicación.

La revisión al programa del curso «Últimas tendencias de investigación en comunicación», que forma parte del Máster en Comunicación Social promovido por la Universidad Pompeu Fabra, en España, permitió constatar varias líneas de estudio de prioridad en Latinoamérica, Estados Unidos, España, Alemania, Francia y Gran Bretaña.

Como tendencias generales de investigación en comunicación del siglo XXI se señalan los efectos de los medios de comunicación, la audiencia como objeto de investigación y la investigación del contenido y de las formas de los referentes mediáticos. Como tendencias teóricas se refieren a las que surgen a mediados del siglo XX (usos y gratificaciones, agenda setting y cultivation theory) y a las de mayor notoriedad durante el siglo XXI (framing, agenda setting, cultuvation theory, third-person effects, usos y gratificaciones). 
Sobre la base de dicho documento, se elaboró la siguiente tabla, donde se mencionan las últimas tendencias y líneas de investigación en comunicación.

\begin{tabular}{|c|c|}
\hline \multicolumn{2}{|c|}{ TENDENCIAS EN INVESTIGACIÓN SOBRE MEDIOS AUDIOVISUALES } \\
\hline Televisión & \\
\hline $\begin{array}{l}\text { Representación } \\
\text { cultural }\end{array}$ & $\begin{array}{l}\text { Estudio de cómo la televisión representa diferentes identidades culturales } \\
\text { y de cómo transmite normas sociales dominantes. }\end{array}$ \\
\hline Recepción & $\begin{array}{l}\text { Cómo se mira la televisión. Etnografías del consumo. } \\
\text { El fenómeno fandom. }\end{array}$ \\
\hline El texto televisivo & $\begin{array}{l}\text { Análisis del lenguaje. Análisis de las estructuras discursivas } \\
\text { y narrativas, y de las estrategias comunicativas. }\end{array}$ \\
\hline Cine & $\begin{array}{l}\text { El texto fílmico. Recepción y efectos del filme sobre el espectador. Fandom } \\
\text { studies. Estudio cognitivista del funcionamiento narrativo del filme. } \\
\text { Estudios culturales, posfeminismo y «gender studies». }\end{array}$ \\
\hline Nuevos medios & $\begin{array}{l}\text { Convergencia. Consumo y patrones de uso. Desigualdades de uso. } \\
\text { Comunidad e identidad en el espacio electrónico. Tiempo y espacio en la } \\
\text { cultura global y la vida cotidiana. }\end{array}$ \\
\hline \multicolumn{2}{|r|}{ TENDENCIAS EN INVESTIGACIÓN SOBRE PUBLICIDAD } \\
\hline \multicolumn{2}{|c|}{$\begin{array}{l}\text { Publicidad y contenido de la comunicación. Publicidad y juegos de ordenador. Publicidad, branding } \\
\text { y comunicación en Internet. Género y publicidad, branding y comunicación. Media placement, } \\
\text { brand placement, relaciones públicas y marketing viral. Temas sociales y publicidad. Interacción } \\
\text { social y publicidad. Organización, publicidad y reputación. Branding. La responsabilidad } \\
\text { corporativa, temas sociales y publicidad. Publicidad en redes sociales; online; móvil. Product } \\
\text { placement. Género, infancia y publicidad. Publicidad y creatividad. Consumidores, empresas, las } \\
\text { marcas y sus relaciones. }\end{array}$} \\
\hline \multicolumn{2}{|c|}{ TENDENCIAS EN INVESTIGACIÓN SOBRE RELACIONES PÚBLICAS } \\
\hline \multicolumn{2}{|c|}{$\begin{array}{l}\text { Las RRPP como una función social. La teoría social como metodología de análisis de la práctica de las } \\
\text { RRPP. Nuevos sujetos activos de las RRPP: estados nación, naciones sin estado, territorios; diplomacia } \\
\text { pública como parte importante de las RRPP. La influencia de la cultura en la práctica profesional. Las } \\
\text { RRPP internacionales. La historia y la historiografía de las RRPP como nuevo ámbito de investigación. }\end{array}$} \\
\hline \multicolumn{2}{|c|}{ TENDENCIAS EN INVESTIGACIÓN SOBRE MEDIOS INFORMATIVOS } \\
\hline Producción & $\begin{array}{l}\text { Medios informativos y rutinas profesionales. Objetividad. Profesionalismo. } \\
\text { Género en la redacción. Convergencia } \\
\text { en la producción de contenidos. }\end{array}$ \\
\hline Contenido & Agenda setting. Framing. Las noticias: discurso e ideología. \\
\hline $\begin{array}{l}\text { Periodismo } \\
\text { y sociedad }\end{array}$ & $\begin{array}{l}\text { Periodismo y democracia. El ciudadano como periodista. } \\
\text { Periodismo y cultura popular. Recepción. }\end{array}$ \\
\hline
\end{tabular}

Tabla 1. Últimas tendencias en investigación en comunicación en el siglo XXI Fuente: programa del curso «Últimas tendencias de investigación en comunicación», Universidad Pompeu Fabra (España) 
Si bien es cierto que los estudios sobre culturas populares han carecido de mayor atención, no debe desestimarse que se trata de un eje temático capaz de ofrecer una multiplicidad de enfoques y de aristas de estudio que no se restringen, exclusivamente, a la producción cultural massmediática. Sus tres líneas de trabajo, propuestas por Jesús Martín-Barbero en 1982, son: de lo popular a lo masivo, de lo masivo a lo popular y usos populares de lo masivo.

Se trata de la capacidad articulatoria de temáticas y de ejes diversos que la investigación culturista permite. «La comunicación y la cultura plantean temáticas complejas y de alcance global, pero, aun así, debe esperarse un tratamiento responsable de esa complejidad, a través de estudios de carácter interdisciplinario o transdiciplinarios» (Lingeri, 2013, en línea).

Valor agregado se añade a la producción epistemológica actual, en la que se hace referencia a los avances tecnológicos que las sociedades alcanzan con el auge de Internet y de las plataformas de producción cultural-comunicativa que emergen y que irradian desde el ciberespacio, dando lugar a nuevas categorías, a saber, cibersociedad, cibercultura y ciberidentidad.

Una problemática social compleja y de carácter multidimensional, como es la comunicación y la información mediada por tecnología digital inteligente, no puede ser abordada si no se toman en cuenta, por ejemplo, variables psicológicas, sociológicas, comunicacionales y educativas (entre algunas otras). Esto exige un cierto grado de diálogo entre distintas disciplinas (León Duarte, 2015, p. 4).

El Séptimo Encuentro Semestral de Metodología Aplicada a la Comunicación, celebrado en México en 2012, fue dedicado a la importancia de la interdisciplinariedad en la investigación y en la práctica de distintos campos; particularmente, de la comunicación. Valiosos fueron los aportes de la académica y comunicóloga Luz María Garay, quien explicó que para hablar de interdisciplinariedad se requiere de la apropiación de conceptos como epistemología, interacción, disciplinas, métodos y comprensión de hechos sociales. 
Se trata de la integración del esfuerzo de los sujetos y de su interés por conocer o por comprender en profundidad, a través de distintas visiones, manteniendo el rigor teórico; de esta manera, las ciencias sociales están ganando terreno a través de investigaciones, pero también a partir de trabajos interdisciplinarios (Garay, citada por Vega, 2012, en línea).

En dicho encuentro, la maestra en antropología e historia, Haydee García, explicó que la interdisciplinariedad es un proceso de comunicación «que atraviesa a la cultura en toda su amplitud»(García, citada por Vega, 2012, en línea), lo que ha permitido asumir otras perspectivas de análisis para explicar los procesos y las prácticas de comunicación en su devenir.

En Latinoamérica,

[...] aun cuando los ejes temáticos de investigación abarcaron distintas esferas, y la aplicación de los presupuestos metodológicos se centraron en el campo comunicológico, estos estudios han enriquecido la mirada sobre diversas aristas del fenómeno comunicativo, lo que ha permitido no solo un enriquecimiento de los saberes de este campo, sino que también le ha aportado información valiosa al resto de las ciencias sociales, en aras de la construcción de esa otra comunicación emergente, fruto del cambio social de que forma parte (Acosta \& Costales, 2013, en línea).

Lo contextual y las múltiples singularidades o complicaciones que emanan de los diferentes sistemas sociales son cuestiones a considerar cuando se realizan estudios desde esta perspectiva.

Pensemos, por ejemplo, en el desarrollo histórico del modelo de comunicación básico donde su tema, objeto y problema mediático analizado presenta, incluso, múltiples niveles de componentes, de relaciones, de conexiones y de escenarios categorizados en orden jerárquico o con base en cierta estructura (León Duarte, 2015, p. 7).

Asimismo, César Ulloa Tapia (2009) asevera que pensar la comunicación en los tiempos que corren implica contextualizar la producción científica en función de los problemas sociales vigentes. 
[...] el calentamiento global, la deshumanización periódica que trae consigo la videovida, la ludopatía, la intolerancia cultural, étnica, religiosa y sexual; el uso de las nuevas tecnologías de la información y comunicación (TIC) bajo un marco instrumental y, estrictamente, rentista, entre otros. De ahí, la importancia de repensar las nuevas líneas de la investigación en comunicación (Ulloa Tapia, 24/7/2009, en línea).

Esto exige que la investigación permita examinar los fenómenos en profundidad, proponer enfoques y modelos teóricos que posean alcances prácticos y que propicien soluciones mediante estrategias o acciones concretas.

Al referirse a la realidad latinoamericana, Martín-Barbero (2008) hace referencia a cómo en el continente la edificación epistemológica se produce en medio de golpes económicos, políticos e institucionales; de la precariedad y la carencia de recursos materiales, técnicos y humanos. Es por eso que «la investigación de la comunicación debe fortalecer uno de sus mayores imperativos: lo social y la sociedad» (Ulloa Tapia, 24/7/2009, en línea). Asimismo,

[...] la investigación de la comunicación ha girado, se ha transformado, se ha resemantizado debido al aparecimiento de las nuevas tecnologías de la información y la comunicación, al uso y la creación de nuevas expresiones culturales, al aparecimiento de innumerables movimientos sociales que tienen particulares maneras de comunicar y [a] los grandes problemas que enfrenta el mundo (Ulloa Tapia, 24/7/2009, en línea).

Vigencia tiene el planteamiento de Paulina Emanuelli (1999), quien apunta:

Es esencial sostener un amplio trazo de libertad intelectual, más allá de disputas disciplinares para posibilitar la generación de conocimientos y de propuestas. Son tiempos en los que es prioritario escuchar, pensar, discutir, compartir, crecer y producir. Es fundamental crear espacios de discusión y de reflexión colectiva y así poder romper con los prejuicios teórico metodológico y alguna puja mezquina de posicionamiento individual. Hoy, más que nunca, los esfuerzos deben orientarse a la permanencia y al desarrollo de temáticas críticas en la universidad pública, justamente, como forma de romper con el dominio académico que responde a la lógica mercantil de la época (en línea). 
En este sentido, las comunidades universitarias y las diferentes organizaciones académicas de investigación que han surgido en los últimos años desempeñan un rol importante; no solo en la formación de profesionales competentes sino también por la excelente capacidad que poseen de producir y de comunicar la ciencia, y de contribuir a la solución de los problemas sociales mediante el diálogo multidisciplinario, donde el fortalecimiento epistemológico y el bien común se consideren ejes transversales y prioritarios.

Investigación en comunicación: grupos y ejes temáticos

En este acápite, haremos alusión a los GT y a los ET de organizaciones como la Asociación Latinoamericana de Investigadores de la Comunicación (ALAIC), la Asociación Española de Investigadores de la Comunicación (AE-IC), la Federación Latinoamericana de Facultades de Comunicación Social (FELAFACS) y la Asociación de Historiadores de la Comunicación (AHC).

ALAIC surge en noviembre de 1978 en Caracas, Venezuela, por iniciativa de destacados estudiosos latinoamericanos de la comunicación, a saber: Antonio Pasquali, Luis Ramiro Beltrán, Jesús Martín-Barbero, Alejandro Alfonso y Marco Ordóñez, entre otros. Desde sus inicios, se propuso fortalecer la investigación científica y articular las comunidades académicas de la región que contaban con programas de comunicación social, periodismo y otras áreas afines. En la actualidad, cuenta con 19 GT [Tabla 2].

Según se expresa en el sitio de la asociación:

Los grupos temáticos (GT) son entidades orgánicas creadas por ALAIC para el intercambio y el trabajo académico de sus miembros en torno a un sub-campo de conocimiento de la especialidad. Como espacios de diálogo, permiten articular intereses e iniciativas para desarrollar líneas y prácticas de investigación en las que un conjunto de asociados participan para poner en común su experiencia y sus trabajos de investigación (ALAIC, Grupos Temáticos ALAIC, en línea). 


\begin{tabular}{|c|l|}
\hline \multicolumn{2}{|c|}{ GRUPOS DE TRABAJO } \\
\hline GT. 1 & Comunicación intercultural y folkcomunicación \\
\hline GT.2 & Comunicación organizacional y relaciones públicas \\
\hline GT.3 & Comunicación política y medios \\
\hline GT.4 & Comunicación y educación \\
\hline GT.5 & Comunicación y salud \\
\hline GT. 6 & Economía política de las comunicaciones \\
\hline GT. 7 & Estudios de recepción \\
\hline GT. 8 & Comunicación popular, comunitaria y ciudadanía \\
\hline GT.9 & Teoría y metodología de la investigación en comunicación \\
\hline GT.10 & Comunicación, tecnología y desarrollo \\
\hline GT.11 & Comunicación y estudios socioculturales \\
\hline GT.12 & Comunicación para el cambio social \\
\hline GT.13 & Comunicación publicitaria \\
\hline GT.14 & Discurso y comunicación \\
\hline GT.15 & Comunicación y ciudad \\
\hline GT.16 & Estudios sobre periodismo \\
\hline GT.17 & Historia de la comunicación \\
\hline GT.18 & Ética, libertad de expresión y derecho a la comunicación \\
\hline GT.19 & Comunicación digital, redes y procesos \\
\hline
\end{tabular}

Tabla 2. Grupos temáticos de ALAIC Fuente: elaboración propia

Asimismo, se explica cómo mediante estos grupos se favorecen el intercambio y el trabajo conjunto de los/as asociados/as, se canalizan intereses de investigación y se proyectan resultados de los esfuerzos académicos. «A través de su actuación, promueven la institucionalización, la vinculación interinstitucional y la actualización permanente del conocimiento en el área y estimulan la cooperación y la integración de sus miembros y de quienes se inician en la actividad» (ALAIC, Grupos temáticos ALAIC, en línea).

Desde este punto de vista, queda claro cómo las redes son organizaciones que tienen la capacidad de favorecer procesos interactivos y de socialización para contribuir a la solución de problemas determinados, que pueden ser investigativos, académicos, sociales, etcétera. 
Al referirse a los GT, Erick Torrico Villanueva (2008) afirma:

Estos grupos son el corazón de ALAIC, pues aglutinan a investigadores de la región, así como a latinoamericanistas de Norteamérica, Europa y Australia que en los congresos y seminarios presentan estudios sobre fenómenos comunicacionales de América Latina para su análisis y su discusión (p. 83).

Mientras que en 1992 existían siete, en la actualidad ALAIC cuenta con diecinueve GT. Este proceso de reorganización ha favorecido el desarrollo de la producción científica en comunicación. Nótese el incremento progresivo de ponencias presentadas en los diferentes congresos. En 1992 se presentaron 50 aproximadamente; en 2004, 523, y en el XIII Congreso Latinoamericano de Investigadores de la Comunicación, efectuado en octubre de 2016 en México, se alcanzó un total de 703 trabajos [Gráfico 1].

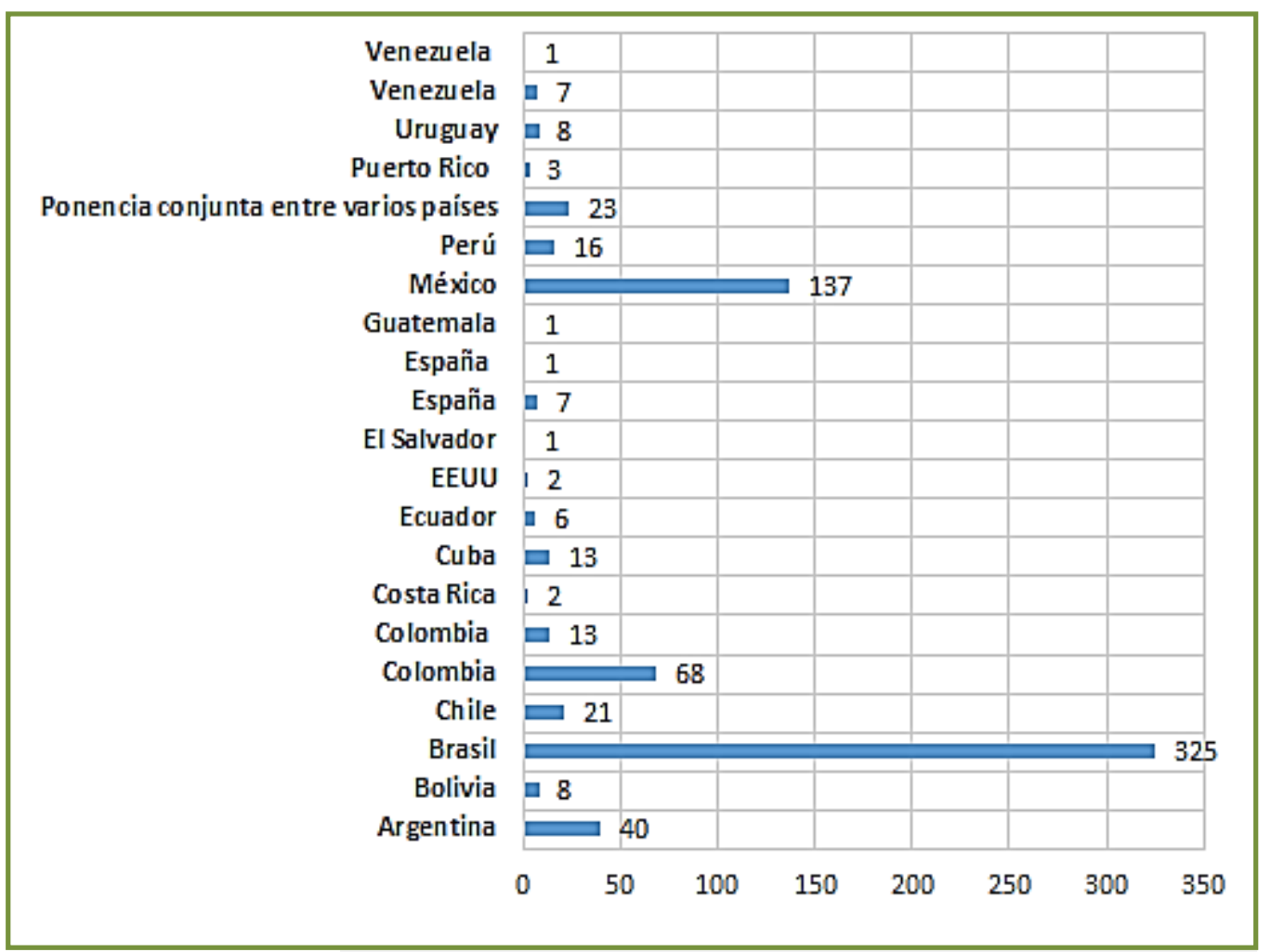

Gráfico 1. Cantidad de ponencias que se presentaron por país en el XIII Congreso de ALAIC de 2016. Fuente: enviado por María Consuelo Lemus Pool, División de Estudios de Posgrado e Investigación, Universidad Autónoma de Tamaulipas (México) 
La AE-IC, organización con sede en la Facultad de Ciencias de la Información de la Universidad Complutense de Madrid, España, establece desde 2014 diferentes Secciones Temáticas y en su interior los diferentes GT [Tabla 3].

\begin{tabular}{|l|l|l|}
\hline & SECCIONES TEMÁTICAS & GRUPOS DE TRABAJO \\
\hline $\mathbf{1}$ & $\begin{array}{l}\text { Teorías y métodos de investigación } \\
\text { en comunicación }\end{array}$ & $\begin{array}{l}\text { Historia de la investigación } \\
\text { en comunicación }\end{array}$ \\
\hline $\mathbf{2}$ & Estudios de audiencia y recepción & - \\
\hline 3 & Estructura y políticas de la comunicación & Comunicación y ciudadanía \\
\hline 4 & Estudios sobre el discurso & Estudios de género y comunicación \\
\hline 5 & Comunicacióny cultura digital & Periodismo e información de calidad \\
\hline 6 & Produccióny circulación de contenidos & \\
\hline 7 & Comunicación estratégica y organizacional & \\
\hline
\end{tabular}

Tabla 3. Secciones temáticas y grupos de trabajo de AE-IC Fuente: elaboración propia

Para ello, la organización española cuenta con el Reglamento de Secciones y Grupos de Trabajo. El artículo 26 de dicho documento habilita «[...] la posibilidad de constituir Grupos de Trabajo en el seno de las Secciones Temáticas de la asociación, entendidos como espacio de coordinación y de intercambio de conocimientos sobre temas específicos y de actualidad o en torno a líneas de investigación emergentes» (AE-IC, 2014, p. 1).

En 2014, se creó el GT «Historia de la investigación sobre comunicación»,

[orientado a] generar un espacio de encuentro académico específico para los/las investigadores/as interesados/as en el estudio histórico de la investigación sobre Comunicación, particularmente centrada en el caso español, aunque no de manera exclusiva, y en el análisis de las características (objetos de estudio, teorías, métodos, etc.) de la investigación más reciente, desde cualquier planteamiento disciplinar (sociología, psicología, antropología, economía, etc., del conocimiento científico). En el primer caso, se trataría de estimular un tipo de trabajo (el de reflexión histórica) escasamente representado en la investigación comunicativa española; y, en el segundo (el análisis de la investigación más reciente), de propiciar la continuidad de una línea de trabajo muy frecuentada en la última década (AE-IC, 2014, Pp. 1-2). 
También ha sido tradición de FELAFACS establecer ET y GT para los diferentes congresos académicos que se efectúan en el continente, los que han variado en correspondencia con los temas que privilegian las universidades participantes y a tono con las corrientes contemporáneas de pensamiento comunicacional latinoamericano. En el XVI Encuentro FELAFACS, que tuvo lugar en Viña del Mar y Valparaíso entre el 18 y el 20 de octubre de 2017, bajo el lema «Re-Evolución: mundos reales y virtuales en la comunicación y el periodismo», se debatieron ponencias que tributaron a tres ET principales [Tabla 4].

\begin{tabular}{|c|l|}
\hline \multicolumn{2}{|c|}{ DENOMINACIÓN DEL EJE DE TRABAJO } \\
\hline ET. 1 & Periodismo: nuevos desafíos, ciudadanías y espacios públicos \\
\hline ET. 2 & Internet: vigilancia y emancipación \\
\hline ET. 3 & Sociabilidad: nuevos dispositivos otros vínculo \\
\hline
\end{tabular}

Tabla 4. Ejes temáticos del XVI Encuentro FELAFACS (2017) Fuente: elaboración propia

Cada ET cuenta con GT formulados en forma de preguntas, a las cuales los/as profesores/as, los/as especialistas y los/as investigadores/as asistentes dan respuesta mediante sus ponencias y sus conferencias en más de cincuenta mesas de trabajo.

Los ejes temáticos definidos para este encuentro tienen por objeto atender a las problemáticas y los desafíos con los que actualmente nos encontramos los profesionales de la comunicación. Asimismo, y por primera vez en un encuentro FELAFACS, dedicaremos un eje temático exclusivamente al ejercicio del periodismo y cómo esta profesión ha debido saber adaptarse a las nuevas formas de consumo medial (Brokordt, 2017, en línea).

Otra evidencia de lo multidisciplinar en la conformación y en el funcionamiento de los GT y los ET de las organizaciones académicas de investigación puede hallarse en el XV Congreso de la AHC, realizado en la ciudad de Porto, en Portugal, los días 14 y 15 de septiembre de 2017. Bajo el tema central «Comunicación y espectáculo», el encuentro incluyó los subtemas: Iberismo y periodismo, Metodologías en la investigación de la historia de la comunicación y Biografías de periodistas. Se trató de una iniciativa conjunta de la AHC y el área de Comunicación de la Universidad de Porto, en la que colaboraron las facultades de Letras, Ingeniería, Bellas Artes y Economía. 
El análisis de los GT y los ET de diferentes convocatorias de eventos científicos sobre comunicación demuestra que no son temáticas fijas las que se priorizan en este tipo de organización sino que se evidencia un intento por la actualización temática a tono con las tendencias emergentes de producción simbólica e infocomunicativa, y en correspondencia con los cambios sociales de la humanidad.

De estos encuentros se generan importantes publicaciones científicas y especializadas, libros, productos comunicativos, memorias que compilan las ponencias de los expositores, artículos para revistas y anuarios, dispositivos virtuales, entre otros.

Los/as asociados/as a las organizaciones pueden afiliarse a un GT específico a partir de sus intereses investigativos, si bien tienen la posibilidad de aportar a varios a la vez en diferentes eventos o encuentros científicos.

También se destacan los llamados Grupos de interés (GI), que «[...] no son fijos; suelen organizarse en función de la demanda de los congresos, especialmente por solicitud de la sede que organiza el Congreso. Algunos logran convertirse en GT (Grupo de Trabajo) que tienen una permanencia a lo largo de los congresos; otros desaparecen y dan pie a la formulación de nuevos GTS» (ALAIC, Grupos de interés, en línea).

Notables han sido los aportes de la Pontificia Universidad Javeriana (PUJ) en Bogotá, Colombia, al contar con los grupos de investigación, «Ciencia de la información, Sociedad y Cultura» y «Comunicación, Medios y Cultura». El segundo se creó en 1997 y figura en la actualidad como uno de los grupos de referencia académica a nivel nacional y regional.

[...] cuenta con producciones de calidad académica, rigor investigativo y pertinencia social, así como con capacidad de gestionar, de organizar y de coordinar redes de investigación y seguimientos de problemáticas de comunicación que tenga proyección social en el país (PUJ, en línea).

Entre sus temáticas de prioridad se encuentran: estrategias de comunicación, políticas de comunicación y cultura, opinión pública, formación ciudadana y medios de comunicación, comunicación en las organizaciones, divulgación de la ciencia y comunicación / educación. 
La RIHC es una organización académica de investigación que cuenta con el respaldo de FELAFACS, además del apoyo y del diálogo permanente que sostiene con representantes, con profesores/as y con investigadores/as de la Asociación Dominicana de Escuelas de Comunicación (ADECOM), la Asociación de Historiadores de la Comunicación (AHC) de España, la Facultad de Ciencias Humanas de la Universidad Autónoma de Baja California (México), la Red de Historia de los Medios (REDHIME) y el Centro de Estudios en Historia / Comunicación / Periodismo / Medios (CEHICOPEME), de la Universidad Nacional de La Plata (Argentina), la Asociación Brasileña de Investigadores en Historia de los Medios (ALCAR), la Facultad de Artes y Comunicación de la Universidad APEC (República Dominicana) y el Grupo Temático sobre Historia de la Comunicación de ALAIC.

Desde su constitución, el 28 de octubre de 2016 durante el desarrollo del VI Encuentro Iberoamericano de Comunicación realizado en Santo Domingo, República Dominicana, la RIHC se propone contribuir al fortalecimiento de la docencia, la investigación y la difusión de conocimientos relacionados con la historia y con la historiografía de la comunicación social; así como favorecer la edificación disciplinar de las ciencias de la comunicación, en consideración de la diversidad sociohistórica y sociocultural de los diferentes países y regiones.

El logro de este propósito requiere, entre otras cosas, del diseño de estrategias que se encaminen a favorecer la investigación desde el enfoque multi/interdisciplinar. Surgen así los GT de la RIHC, siguiendo la tendencia que prevalece en distintas organizaciones y asociaciones profesionales que abordan el campo de la comunicación, a la hora de enfocar los grupos temáticos y de considerar la coexistencia de campos ya configurados y de larga data con otros más emergentes.

Las valoraciones y las contribuciones de la colectividad fueron imprescindibles para la conformación de los GT. Se trata de un proceso de participación y de intercambio, en el que más de 90 asociados/as tuvieron la posibilidad de emitir criterios, de corregir y de ofrecer ideas que contribuyan a organizar las líneas y las sublíneas de estudio privilegiadas por la organización. Desde este punto de vista, se favorece la concepción multidisciplinaria y heterogénea de los GT y se logra que los/as asociados/as y los/as investigadores/as interesados en las actividades de la RIHC encuentren en la red un espacio al cual tributar. 
Oportunas fueron las consultas realizadas al doctor Gilberto Eduardo Gutiérrez, profesor de la Pontificia Universidad Javeriana de Bogotá (Colombia) y coordinador principal del GT «Historia de la comunicación» de ALAIC, y al doctor César Luis Díaz, director del CEHICOPEME, centro que auspicia la Universidad Nacional de La Plata (Argentina).

Las propuestas iniciales fueron realizadas por varios miembros de la junta directiva de la red, quienes sugirieron un total de 17 GT. Luego, tras el proceso de consulta colectiva, se consideró la necesidad de privilegiar diez temáticas y de insertar a las siete restantes como subtemas.

Los GT de la RIHC tienen como objeto central los estudios históricos y la producción historiográfica de la comunicación, a nivel internacional, continental, nacional, regional y local. De igual manera, puede tratarse de investigaciones ocupadas en examinar fenómenos o producciones contemporáneas, considerándose que la historia $y$, en particular, la historia de la comunicación no concierne exclusivamente al pasado, sino que es construida por los sujetos sociales en sus relaciones cotidianas.

\begin{tabular}{|c|c|}
\hline \multicolumn{2}{|r|}{ DENOMINACIÓN DE LOS GT } \\
\hline GT. 1 & Historia del campo académico e investigativo de la comunicación \\
\hline GT. 2 & Historia de los medios y del periodismo \\
\hline GT. 3 & $\begin{array}{l}\text { Historia y comunicación organizacional / publicidad / } \\
\text { comunicación visual / relaciones públicas }\end{array}$ \\
\hline GT. 4 & Estudios históricos sobre producción audiovisual \\
\hline GT. 5 & Comunicación, culturay sociedad \\
\hline GT. 6 & $\begin{array}{l}\text { Historia y comunicación política / ciudadanía, participación / } \\
\text { movimientos sociales }\end{array}$ \\
\hline GT. 7 & Arqueología de la comunicación local / regional / nacional \\
\hline GT. 8 & Subjetividades, microhistoria e historias de vida \\
\hline GT. 9 & Memoria y medios \\
\hline GT. 10 & Enseñanza de la historia de la comunicación \\
\hline & $\begin{array}{r}\text { Tabla 5. Grupos Temáticos de la Red Internacional } \\
\text { de Historiógrafos de la Comunicación (RIHC) } \\
\text { Fuente: elaboración propia }\end{array}$ \\
\hline
\end{tabular}




\section{GT.1 | Historia del campo académico e investigativo de la comunicación}

Privilegia la historia de la enseñanza en comunicación, incluyendo las carreras de periodismo, ciencias de la información, publicidad, diseño y otras disciplinas afines que se estudian en varias universidades del mundo. Admite el análisis histórico de planes, modelos, programas, metodologías y políticas educativas en comunicación.

Se examina y se reflexiona desde la perspectiva histórica acerca de los distintos planteamientos disciplinares, su incidencia en la producción científica y en el propio campo académico de la comunicación (la sociología, la filosofía, la historia, la psicología, la antropología, la economía, etc.).

Asimismo, apoyados en el GT «Historia de la investigación sobre comunicación» de la AE-IC, se establecen otros ejes o sublíneas, a saber:

- Análisis de la producción científica: de objetos de estudio, métodos, teorías, prácticas, modelos, enfoques y perspectivas, categorías y dimensiones, líneas de investigación, ideas, autores, escuelas, influencias, adscripciones epistemológicas y teórico-metodológicas.

- Estudios sobre la comunidad científica: asociaciones, redes de investigación, grupos y sociedades científicas, perfiles académicos, etcétera.

- Análisis del contexto institucional: centros de investigación, regulaciones normativas, políticas científicas, revistas especializadas, anuarios científicos, programas de fomento y de financiación, etcétera.

Generalmente, estos temas se encuentran asociados al propio campo académico, desde donde se promueve la investigación y la comunicación científica.

\section{GT. 2 | Historia de los medios y del periodismo}

Comprende el tipo de investigación tradicional en comunicación, efectuada también desde ciencias como la historia y la sociología. ${ }^{2}$

La historia de los medios de comunicación y del periodismo permite que se realicen estudios de caso y se examinen sistemas o subsistemas mediáticos (la prensa, el cine, la radio, la televisión y los medios digitales). Se añaden, la construcción transdisciplinar e histórica de las ideologías profesionales del periodismo, los estudios históricos de recepción, las instancias de producción, de distribución y de recepción de los mensajes periodísticos, así como los estudios en clave comparativa (máxime si se contemplan países de la región, normativas que regulan las políticas de medios, etc.). 
El análisis sociohistórico de los medios se enmarca en las fases de producción, de circulación y de recepción. A esto se suman análisis diacrónicos (relaciones entre los medios que se estudian y los que los anteceden) y análisis sincrónicos (relaciones entre los medios que se estudian y los medios contemporáneos o los procesos culturales que les impactan).

El componente ideológico y los discursos políticos, económicos, comerciales, culturales y sociales en la producción infocomunicativa massmediática y en el ejercicio del periodismo se insertan en este caleidoscopio, y bien pueden constituir subtemas para investigaciones independientes y heterogéneas. Asimismo, se examinan las relaciones de interdependencia entre sistemas mediáticos y sistemas sociales, para lo cual es necesario auxiliarse de los aportes que ofrecen la Teoría social de la comunicación (Martín Serrano, 1977, 1986) y la Metodología histórico estructural para el análisis de los medios (Sánchez Ruiz, 1991).

\section{GT.3 | Historia y comunicación organizacional / publicidad / comunicación visual / relaciones públicas}

Se centra en la investigación histórica de la comunicación organizacional, en la que se inscribe la historia de las relaciones públicas, la publicidad, la comunicación visual, la fotografía y el análisis histórico de cada uno de los productos, discursos, estrategias, campañas y procesos o tendencias de producción de contenido desde esta perspectiva; sea la presencia institucional en el ámbito mediático, o los discursos que las instituciones posicionan en el espacio de lo público por el recurso a otros soportes (escriturales, gráficos orales). Las relaciones históricas de interdependencia entre el sistema de comunicación institucional (empresas, corporaciones, organismos, etc.) y el sistema social se constituyen como un ámbito de oportunidades empíricas, sobre el cual pueden realizarse estudios integrados, multidisciplinarios, comparados, holísticos y exámenes particulares.

\section{GT. 4 | Estudios históricos sobre producción audiovisual}

Aborda lo referente a televisión y cine, a programación y secciones de programas, a géneros y formatos (noticieros, documentales, espectáculos televisados, series, telenovelas, filmes), a estilos y lenguajes audiovisuales, y a los procesos de producción audiovisual (estudios comparados y análisis sobre su evolución). No se trata de su historia como medios, sino del examen diacrónico e interpretativo de los sistemas de programación y de las dinámicas de producción audiovisual; 
aspectos que, generalmente, la historia de los medios no resuelve, toda vez que, además de la descripción, exige privilegiar el análisis hermenéutico / interpretativo para comprender las interdependencias con respecto al sistema social.

\section{GT. 5 | Comunicación, cultura y sociedad}

Se estudian, aquí, la producción simbólica pública (no massmediática), los discursos, los soportes simbólicos, las prácticas culturales y los espacios de socialización que forman parte de la vida cotidiana y que se convierten en portadoras y en generadoras de identidades y de subjetividades. ${ }^{3}$

Admite el examen de las políticas educativas y de la comunicación en ámbitos escolarizados; inter, multi y pluriculturalidad; arte, semiótica, estudios lingüísticos; religiosidad y espiritualidades; ciudad, migración y demografía; folclor y cultura popular; espacios públicos y de sociabilidad, gremios, asociaciones, prácticas comunicativas no mediáticas, etcétera. ${ }^{4}$

\section{GT. 6 | Historia y comunicación política / ciudadanía, participación / movimientos sociales}

Se aborda la historia de la propaganda en todas sus expresiones y sus manifestaciones (política, ideológica / filosófica, religiosa), unido a las tendencias contemporáneas de comunicación política por el recurso a la oralidad y a la tecnología (sitios de redes sociales, blogs, páginas web institucionales). Lo referente a las prácticas contrahegemónicas emergentes que se advierten en la modernidad y en la posmodernidad, a las prácticas de comunicación encaminadas a la cimentación o al fortalecimiento de la ciudadanía, a la participación social, y lo simbólico presente en los movimientos sociales y populares con el afán de emanciparse y de posicionar sus sistemas de ideas y de valores.

\section{GT. 7 | Arqueología de la comunicación local / regional / nacional}

Pone el acento en los estudios históricos de la comunicación en entornos no muy privilegiados por la investigación de nuestra ciencia: comunidades rurales y semirurales, sectores indígenas y campesinos, municipios, barrios. La historia de la comunicación pública regional / local y su interdependencia con los sistemas sociales. Las mediaciones y el examen de las configuraciones y las relaciones que se dan entre la trilogía local-regional-nacional. 


\section{GT. 8 | Subjetividades, microhistoria e historias de vida}

Una agenda que procura la vuelta a la historia de los sujetos / actores sociales históricamente preteridos de la historia tradicional y oficial; que se detiene en los rostros anónimos y en sus travesías singulares, a menudo desde la alteridad o a la sombra de configuraciones hegemónicas; donde se funde lo mediático y lo popular como objetivación en el espacio público más estructurado o bien en el espacio público local, y como expresión de resistencia identitaria. Se aplica también al microespacio o al microcampo de las organizaciones, cualquiera sea su tipología.

\section{GT. 9 | Memoria y medios}

Se trabaja el modo en el que los medios tradicionales y los más actuales contribuyen a la afirmación o al quebrantamiento de las culturas nacionales, a los procesos de reproducción de las identidades culturales, a la imbricación de lo trasmedia en la generación de contenidos que tributan a la preservación de la memoria (presencia de la memoria histórica y cultural, la identidad, lo popular, lo tradicional, las culturas ancestrales y lo autóctono en la producción de contenido simbólico massmediático), el género testimonial histórico en la radio y en la televisión, el cine testimonial y el documental. Cómo las plataformas infocomunicativas por el recurso a la Internet ofrecen espacios para contribuir a la preservación de la memoria y de la herencia cultural, etcétera.

\section{GT. 10 | Enseñanza de la historia de la comunicación}

Comprende en su concepción a la enseñanza de la historia (de la comunicación y las ciencias de la información, de los medios, del periodismo, la publicidad, las relaciones públicas, la propaganda, el diseño, etc.). Su propia concepción admite que se constituya como un GT independiente, si bien pueden existir diálogos con otros. Admite análisis relacionados con el estado actual de la enseñanza de la historia de la comunicación; de las universidades y de los países donde se ha otorgado cierto grado de importancia a este campo y de las carreras en las que se imparten asignaturas relacionadas con la historia de la comunicación (las materias fundamentales, los programas, las guías de estudio, las bibliografías básicas y complementarias para la docencia, etcétera). 
Entender estos temas como GT es asumirlos como líneas de investigación a las cuales tributan los/as asociados/as con sus estudios, al tiempo que se favorece la participación en los diferentes espacios de la RIHC. Cada asociado/a es libre de decidir en qué GT se inscribirá, si bien puede aportar a varios con sus investigaciones.

Los resultados de investigación de los/as asociados/as a la red, tal como funciona en otras organizaciones, e incluso en las revistas científicas, constituyen propiedad de los/as autores/as. Sin embargo, si se trata de contribuciones que tributan a las líneas y a los grupos temáticos de la red, y se socializan en los congresos, en el sitio, en actas, en memorias o en la revista científica de la organización, se trata de un trabajo conjunto y cooperativo.

La creación y el funcionamiento de los GT es prioridad de la red, con la perspectiva de organizar las sesiones científicas de los congresos y de motivar a la realización de investigaciones históricas o historiográficas que no se restrinjan al estudio de los medios; en pos de que los estudiosos de la publicidad, las relaciones públicas y de otros campos de la comunicación social también encuentren aquí un espacio de oportunidades.

Como se trata de una red académica de investigación de carácter internacional, cada GT cuenta con un coordinador y dos vicecoordinadores, procedentes de universidades y de centros de estudio de diferentes países de Latinoamérica, el Caribe y Europa.

Es importante que se reconozca que los GT no pueden ser grupos que se organicen solo en el contexto de los congresos, sino que deben permitir que se agrupen investigadores/as (profesores/as, especialistas y estudiantes), se generen intercambios, se socialicen y se compartan experiencias, se promuevan publicaciones científicas o de otro tipo y se desarrollen e implementen proyectos. Por esa razón, se organizan de forma flexible y dinámica, y cada una de las iniciativas es resultado del trabajo cooperativo y multidisciplinario entre las diferentes universidades y la RIHC. De allí que resulta de mucha utilidad que se generen convenios de cooperación en materia de docencia y de investigación.

La conformación de los diferentes GT permite que los aportes y las iniciativas no sean de interés exclusivo para investigadores/as (profesionales y estudiantes) de comunicación social o periodismo. Por el contrario, se promueve un movimiento científico multidisciplinario resultado de los trabajos y las iniciativas procedentes de otros campos disciplinares; a saber, sociología, psicología, filosofía, historia / historiografía, pedagogía y ciencias de la educación, antropología, derecho, ciencias políticas, etcétera. 
El análisis de la ficha donde consta el registro de asociados/as da cuenta de cuan diversa es la conformación de la membresía de la RIHC en cuanto al perfil de sus miembros, que no se limita a profesores/as o a especialistas de la comunicación social —máxime cuando la red está abierta a profesionales de ramas afines que encuentren en ella una oportunidad cercana a sus líneas de investigación - sino que se caracteriza por la diversidad sociodemográfica y profesional. Los campos o disciplinas a las cuales pertenecen los miembros de la red comprenden: ciencias de la comunicación, periodismo, ciencias de la información, publicidad y diseño, ciencias históricas, ciencias de la educación y ciencias sociales (Mena Méndez, Segarra-Saavedra \& Muñoz Uribe, 2017).

Se agrega a lo anterior que no solo los/as asociados/as tienen la posibilidad de participar en los eventos (simposios, concursos, congresos) y en las publicaciones científicas promovidas por la red, sino que otros/as profesores/as, investigadores/as y estudiantes de cualquier especialidad, país o institución podrán favorecerse y aportar, siempre que sus iniciativas y sus trabajos se ajusten a la esencia de la organización y respondan a sus GT.

\section{Conclusiones}

La perspectiva multi/interdisciplinar en los estudios de comunicación social ha constituido una tendencia que ha ganado en espacio y en configuración durante las últimas décadas. La pluralidad en la visualización de las prácticas y los procesos de comunicación, unido a la diversificación de temas y de problemas de interés, son cuestiones que van de la mano.

La consolidación de redes y de asociaciones académicas de investigación en comunicación en América Latina ha provisto de nuevos horizontes en los campos de estudio del área, en relaciones de inter y de multidisciplinariedad. Esta expansión del campo ha devenido en el fomento de la calidad investigativa, en el fortalecimiento de la divulgación de los alcances y los resultados de las investigaciones, en el intercambio académico y en la investigación colaborativa. Y la relación entre dichos horizontes ampliados y la apertura de nuevos GT es de mutuo enriquecimiento.

La tendencia creciente al interior del campo científico, sobre todo en lo concerniente a escenarios de debate público (congresos, foros, asociaciones profesionales) y a la organización de la investigación desde enfoques y desde prácticas inter / multi y transdisciplinares da cuenta, a la postre, de que la agenda social apremia articulaciones 
y consensos para lo cual ello constituye un paso significativo, incluso para promover los diálogos entre los liderazgos académico científicos y los políticos, y la incidencia en la formulación de políticas públicas.

Los congresos, los seminarios y los simposios son los espacios que las organizaciones académicas de investigación destinan para favorecer el funcionamiento de los GT, mediante el intercambio y la socialización de experiencias procedentes de diferentes países y regiones. No solo los miembros tienen la posibilidad de participar, también profesores/as, investigadores/as, profesionales y estudiantes que tengan interés en las mesas o en las comisiones para los debates.

Tratándose la Red Internacional de Historiógrafos de la Comunicación de una organización joven, y que se encuentra en proceso de organización en su primer año constitutivo, queda pendiente dar continuidad a este trabajo para futuras publicaciones donde sea lógico realizar un análisis de mayor profundidad acerca del funcionamiento de cada GT, sus resultados y sus impactos.

Aportar al campo epistemológico de la comunicación desde prácticas empíricas emergentes, como lo es la historia de la comunicación, resulta a todas luces fructífero, toda vez que se trata de propiciar la comprensión, la reflexión y el análisis de fenómenos comunicativos en diálogo con el sistema social, interpretándose una serie de procesos económicos, políticos, sociales y culturales para comprender cómo la comunicación transversaliza cada espacio en el cual los sujetos construyen sus relaciones históricas y cotidianas.

\section{Referencias}

Acosta Damas, M. y Costales, Z. (2013). Una comunicación emergente en América Latina. La Jiribilla. Revista de cultura cubana, XII(657). Recuperado de http://www.epoca2.lajiribilla.cu/articulo/6439/una-comunicacion-emergente-enamerica-latina

Asociación Española de Investigadores de la Comunicación (AE-IC) (2014). Propuesta de constitución del grupo de trabajo de la AE-IC. Historia de la investigación sobre comunicación. Bases reglamentarias. Madrid, España: Universidad Rey Juan Carlos. 
Asociación Latinoamericana de Investigadores de la Comunicación (ALAIC). Grupos

Temáticos ALAIC. Recuperado de https://www.alaic.org/site/grupos-de-trabalho/

Asociación Latinoamericana de Investigadores de la Comunicación (ALAIC). Grupos de interés. Recuperado de https://www.alaic.org/site/grupos-de-interes-gi/

León Duarte, G. (2015). La práctica interdisciplinaria aplicada al estudio de la comunicación y la información mediada por tecnología digital. Revista Iberoamericana de las Ciencias Sociales y Humanísticas, 4(8). Recuperado de https://www.ricsh.org.mx/index.php/RICSH/article/view/56

Lingeri, D. G. (2013). El campo de la comunicación: ¿disciplina, interdisciplina o transdisciplina? Una mirada crítica sobre la tensión comunicación-cultura. Revista Argentina de Humanidades y Ciencias Sociales, 11(2). Recuperado de https://www.sai.com.ar/metodologia/rahycs/rahycs_v11_n2_05.htm

Martín-Barbero, J. (1982). Cultura popular y comunicación de masas [conferencia]. Primer Foro Internacional sobre Comunicación y Poder, Lima, Perú.

Martín-Barbero, J. (2008). De los medios a las mediaciones. Comunicación, cultura y hegemonía. La Habana, Cuba: Pablo de la Torriente.

Martín Serrano, M. (1977). La mediación social. Madrid, España: Akal.

Martín Serrano, M. (1986). La producción social de la comunicación. Madrid, España: Alianza.

Sánchez Ruiz, E. (1991). Apuntes sobre una metodología histórico-estructural con énfasis en el análisis de los medios de difusión. Comunicación y Sociedad, (10-11).

Torrico Villanueva, E. (2008). ALAIC y la investigación comunicacional latinoamericana en tiempos de redefinición utópica. Revista Latinoamericana de Ciencias de la Comunicación, (8-9), 78-86. Recuperado de https://www.alaic.org/revista/index.php/alaic/article/view/58/56 
Brokordt, H. (2017). FELAFACS 2017 define Ejes temáticos del XVI Encuentro Latinoamericano de Comunicación Social. Recuperado de http://felafacs.org/news/felafacs-2017-define-ejes-tematicos-del-xvi-encuentrolatinoamericano-de-comunicacion-social/

Dávila Lorenzo, M. y Lorenzo Suárez, I. (2016). La interdisciplinariedad: un enfoque necesario entre las disciplinas Teoría e Investigación en Comunicación y Comunicación Institucional. Conrado, 12(55), 63-67. Recuperado de https://conrado.ucf.edu.cu/index.php/conrado/article/view/359

Emanuelli, P. (1999). ¿Instituciones públicas y/o privadas? El lugar de la investigación crítica argentina de la comunicación en los años 2000. Revista Latina de Comunicación Social, (20). Recuperado de http://www.revistalatinacs.org/a1999eag/61pau.htm

Ferré Pavía, C. (2008). Historia de la comunicación: una reflexión historiográfica. Portal de la Comunicación, (s/n).Recuperado de http://www.portalcomunicacion.com/uploads/pdf/42_esp.pdf

Mena Méndez, D.; Segarra-Saavedra, J. y Muñoz Uribe, J. (2017). La Red Internacional de Historiógrafos de la Comunicación (RIHC), un espacio de cooperación e integración para potenciar la docencia, investigación y difusión científica en comunicación. Revista de la Asociación Española de Investigación de la Comunicación, 4(8), 17-26. Recuperado de http://www.revistaeic.eu/index.php/raeic/article/view/131

Pontificia Universidad Javeriana (PUJ) (2017). Comunicación, Medios y Cultura. Recuperado de http://comunicacionylenguaje.javeriana.edu.co/investigacionpublicaciones/grupos-investigacion

Ulloa Tapia, C. (24 de julio de 2009). Nuevas líneas de investigación en comunicación [Entrada de blog]. Agencia latinoamericana de información. Recuperado de https://www.alainet.org/es/active/31936

Últimas tendencias de investigación en comunicación (programa de curso). Máster en Comunicación Social, Universidad Pompeu Fabra. Barcelona, España. 
Vega, V. (8 de mayo de 2012). Debe ser interdisciplinaria la investigación en comunicación [Entrada de blog]. AUNAM. Agencia universitaria de noticias. Recuperado de http://aunamnoticias.blogspot.com/2012/05/debe-ser-interdisciplinaria-la.html

\section{Sitios institucionales}

Asociación Española de Estudios de Mercado, Marketing y Opinión (AEDEMO)

Asociación Española de Investigadores de la Comunicación (AE-IC)

Asociación de Historiadores de la Comunicación (AHC)

Asociación de Investigadores en Relaciones Públicas (AIRP)

Asociación Latinoamericana de Investigadores de la Comunicación (ALAIC)

Asociación para la Investigación de Medios de Comunicación de España (AIMC)

Centro de Estudios en Historia / Comunicación / Periodismo / Medios (CEHICOPEME)

Red de Historia de los Medios (REDHIME)

Red de Investigación en Comunicación Comunitaria, Alternativa y Participativa (RICCAP)

Red Iberoamericana de Investigadores en Publicidad

Federación Latinoamericana de Facultades de Comunicación Social (FELAFACS)

\section{Notas}

1 Entrevista realizada por los autores para el trabajo «La Red Internacional de Historiógrafos de la Comunicación (RIHC), un espacio de cooperación e integración para potenciar la docencia, la investigación y la difusión científica en Comunicación», en proceso de elaboración.

2 Queda claro que luego del surgimiento de las carreras de comunicación social el examen histórico de lo massmediático se expandió y se diversificó en cuanto a tipologías de estudio, categorías y dimensiones.

3 Algunas de las prácticas culturales a estudiar pueden ser: festejos populares, carnavales, parrandas, procesiones y otros rituales religiosos, juegos, costumbres y tradiciones culinarias, el pregón y los comerciantes ambulantes, lectura de tabaquería, manifestaciones teatrales en espacios públicos, actuaciones periódicas de retretas y de otras modalidades o conjuntos musicales, charangas, comparsas, jolgorios campesinos en contextos urbanos, rurales y semirurales, tertulias y algunas prácticas que por el recurso a la oralidad llegan a alcanzar connotación en ámbitos comunitarios específicos, como pueden ser los cuenteros y los juglares. 
4 Resulta fructífero para este GT la realización de estudios sobre espacios urbanos y contextos donde se producen las prácticas comunicativas / culturales (plazas, paseos, mercados, salones, prados y otros sitios e instituciones), los elementos intervinientes (vestuario y decoración asociada), los actores concurrentes y los roles comunicativos, los tipos de mensajes y los soportes utilizados (escritural, grafico, visual, oral o gestual); las actividades lúdicas y festivas, las mediaciones de género, etarias, de estatus social-jerárquicas, políticas, ideológicas, culturales, referenciales, individuales, económicas, sociales, étnicas y sociohistóricas. 